\title{
IMPACTO DEL INTERNET GLOBAL "STARLINK"
}

AUTORES: Sara Fernanda Cárdenas Reyes ${ }^{1}$

Karla Victoria Mero Franco ${ }^{2}$

Alexander David Chancay Sancan ${ }^{3}$

María Isabel Chancay Tumbaco ${ }^{4}$

Mario Javier Marcillo Merino ${ }^{5}$

\section{DIRECCIÓN PARA CORRESPONDENCIA: (cardenas-sara4113@unesum.edu.ec )}

Fecha de recepción: 11/03/2021

Fecha de aceptación: 22/12/2021

\section{RESUMEN}

En resumen, el articulo tuvo como propósito dar a conocer el impacto global que logro Starlink, el servicio se instaló para pocos territorios privilegiados, según la compañía se logró buenos resultados en la latencia como en la velocidad de descarga. Los métodos usados se refieren a una investigación basada en la experimentación u observación, conducida generalmente a responder una pregunta específica o hipótesis. Como resultados este servicio no pretende competir con las conexiones 5G o la fibra que son las redes disponibles en los actuales momentos, si no complementar ambas, Starlink no se basa en la velocidad si no en el abastecimiento del internet. Finalmente sabemos que el internet satelital tiene un enfoque diferente a lo tradicional, ya que busca la disponibilidad en sectores donde nunca antes existía dicha posibilidad de acceder al internet. Como usuarios obsesionados con la tecnología y en especial del internet, esperamos que Starlink tenga muchos éxitos.

PALABRAS CLAVE: latencia; velocidad; disponibilidad; sectores; internet.

\section{IMPACT OF THE GLOBAL INTERNET "STARLINK"}

\section{ABSTRACT}

\footnotetext{
${ }^{1}$ Quinto semestre. Tecnología de la Información. Ciencias Técnicas. Universidad Estatal del Sur de Manabí Pajan, Litoral y Ecuador. E-mail: cardenas-sara4113@unesum.edu.ec

${ }^{2}$ Quinto semestre. Tecnología de la Información. Ciencias Técnicas. Universidad Estatal del Sur de Manabí. Montecristi, Litoral y Ecuador. E-mail: Mero-karla7537@unesum.edu.ec

${ }^{3}$ Quinto semestre. Tecnología de la Información. Ciencias Técnicas. Universidad Estatal del Sur de Manabí. Jipijapa, Litoral y Ecuador. E-mail: chancay-alexander6899@unesum.edu.ec

${ }^{4}$ Quinto semestre. Tecnología de la Información. Ciencias Técnicas. Universidad Estatal del Sur de Manabí. Jipijapa, Litoral y Ecuador. E-mail: chancay-maria5412@unesum.edu.ec

${ }^{5}$ Ingeniero en Sistema. Docente de la UNESUM. Universidad Estatal del Sur de Manabí. Jipijapa, Litoral y Ecuador. E-mail: mario.marcillo@unesum.edu.ec
} 
In summary, the article was intended to publicize the global impact that Starlink achieved, the service was installed for few privileged territories, according to the company, good results were achieved in latency as well as in download speed. The methods used refer to an investigation based on experimentation or observation, generally conducted to answer a specific question or hypothesis. As a result, this service does not intend to compete with the $5 \mathrm{G}$ connections or the fiber that are the networks available at the moment, if not to complement both, Starlink is not based on speed but on the supply of the internet. Finally, we know that the satellite internet has a different approach to the traditional one, since it looks for availability in sectors where such possibility of accessing the internet never existed before. As users obsessed with technology and especially the internet, we expect Starlink to be very successful.

KEYWORDS: latency; speed; availability; sectors; internet.

\section{INTRODUCCIÓN}

El proyecto Starlink de Elon Musk pretende conectar al mundo con su inmensa constelación de satélites que proporcionará internet de banda ancha a todo el planeta.

En los actuales momentos este servicio está disponible para unos pocos privilegiados, según la compañía se logró buenos resultados en la latencia como en la velocidad de descarga. En total se pondrán más de 42.000 satélites en órbita a una distancia de 328 y 580 kilómetros por encima de la tierra consiguiendo una cobertura global.

Elon Musk al contar con su propia empresa espacial (SpaceX) cuenta con la ventaja de poder desplegar una mayor cantidad de satélites de Starlink a un menor costo al poder reutilizar los cohetes de sus compañías, esto permite que el costo de las antenas de Starlink tenga un menor costo y seas más accesible a todos los consumidores (Disney \& Wallace, 1982), (McDowell, 2020).

Las problemáticas posibles en el futuro: 42.000 satélites puesto en órbita causarían una cantidad de desechos espaciales muy grandes eso sin contar con las posibles colisiones que se podrían dar al tener una cantidad tan grandes de satélites circulante en la órbita terrestre.

\section{DESARROLLO}

Las conexiones de internet por satélite jamás han terminado de cuajar, sin embargo, Elon Musk desea convertirlas en algo muchísimo más común de lo cual lo son ahora. Starlink, su ambicioso plan al respecto, expone tener en órbita alrededor de 12.000 satélites en el futuro. El problema es que aquellas conexiones satelitales continuamente estuvieron bastante limitadas, más que nada ahora que gozamos de conexiones de banda ancha excepcionales. Lo cual parece claro es que Elon Musk no desea hacer enfadar a las gigantes operadoras de telefonía e internet, su servicio dice que no compite con la fibra o las conexiones 5G, sino que complementa las dos ofertas (YONEMOCHI et al., 2002).

Lo cierto es que las conexiones satelitales continuamente estuvieron en especial enfocadas a ámbitos rurales, y al parecer aquel es el primer objetivo de SpaceX y el lanzamiento del servicio Starlink: con él va a ser viable ofrecer servicio en regiones que poseen difícil ingreso a redes estáticas o inclusive redes móviles.

16 UNESUM-Ciencias. Publicación cuatrimestral. Vol. 6, Año 2022, No. 1 (Enero - Abril) 
SpaceX ha anunciado que está intentando encontrar beta-testers para esta constelación de satélites, y se espera que proporcione aquel servicio de manera preliminar en escasas semanas para un número reducido de usuarios en dicha primera etapa. El interés y la expectativa es monumental, sin embargo, Elon Musk ha indicado que por ahora las pruebas se centrarán en "latitudes altas" como la de Seattle en USA, aun cuando parece que en otros territorios como Alemania además son candidatos a poder probar el servicio. Se espera que el lanzamiento comercial de Starlink se genere en USA anterior a que acabe el año, y si todo el despliegue sigue a este ritmo el servicio va a estar disponible en básicamente cualquier parte de todo el mundo durante 2021 (Bromberg et al., 2011).

\section{¿Qué es Starlink?}

Starlink es un proyecto que surgió en la compañía de vehículos aeroespaciales SpaceX, con el objeto de producir una constelación de satélites de órbita baja para proporcionar servicio de Internet internacionalmente. Este servicio debería caracterizarse por ser de banda ancha, cerca de 1 Gbps de descarga, y de baja latencia entre 7 y 30 ms para competir de manera directa con la fibra óptica. El desarrollo de dichos satélites empezó en el año 2015, obteniendo los requisitos legales para los lanzamientos en 2017, y en 2018 la autorización gubernamental de USA. Así la constelación inició a tener forma el 23 de mayo de 2019 una vez que se lanzaron al espacio los primeros 60 satélites Starlink versión 0.9 (Kassas et al., 2021).

\section{Propósitos de Starlink}

La red Starlink ha nacido con el primordial objetivo de proporcionar conexión a internet de banda ancha a cualquier región geográfica de todo el mundo. Uno de los más importantes inconvenientes que descubren varios habitantes canadienses y EEUU es su inviabilidad de tener Internet por habitar en sitios rurales bastante alejados. SpaceX surgió destinados a privatizar los lanzamientos espaciales y ser independiente en la carrera por llegar a Marte en las siguientes décadas. Starlink, por consiguiente, va a ser un plan para financiar los costos de averiguación y desarrollo de vehículos espaciales para el mantenimiento de nuestra red y para la llegada al mundo rojo (Chowdhury et al., 2003).

\section{Disponibilidad de Starlink}

La nutrida presencia de satélites surcando el espacio, ha propiciado que usuarios de determinadas regiones de EEUU y Canadá ya logren probar el servicio en etapa beta. Ni mucho menos hablamos de una etapa definitiva de venta. Cualquier persona de nosotros mismos tenemos la posibilidad de suscribirnos en el portal de Starlink para que SpaceX avise en qué momento habrá disponibilidad en nuestra ubicación. Se implica que para este año 2021 ya se encontrará disponible para otras regiones diversas a América del norte. Tras suscribirse en la web, los usuarios que tengan ingreso y deseen participar en la etapa de pruebas recibirán una antena en su vivienda. Para ponerla en uso van a ser tan sencillo como conectarla a la corriente y orientarla hacia el cielo (Bratspies, 2002)

\section{Velocidad y latencia fase de pruebas Starlink}

Se han publicado los primeros datos de prestaciones de la red Starlink para un definido número de usuarios que se hallan en etapa beta. Lo cierto es que los valores no permanecen nada mal a 
una fase todavía temprana para el servicio. En las primeras cifras que está arrojando la red en el sector de Seattle y Los Ángeles, las velocidades son de 50 Mbps para la descarga y alrededor de los 10 Mbps para la subida. Dichos no son justamente los resultados prometidos en un inicio, debemos comprender que la flota de satélites sigue siendo poca en términos de cercanía entre unos y otros. Más de 200 pruebas llevadas a cabo en diversos puntos de vista estadounidense las cifras que permanecen entregando son de entre 11 y $60 \mathrm{Mbps}$ de descarga con una media cerca de los $30 \mathrm{Mbps}$, mientras tanto que la descarga va a partir de los 5 a 18 Mbps con una media de 6 Mbps. Sin lugar a dudas es una rapidez apta para consumir contenido multimedia en alta definición y descargas subjetivamente rápidas al grado de otros servicios por satélites teniendo presente que esta red todavía está en etapa inicial (Segarra et al., 2001).

\section{Funcionamiento de los satélites}

En el espacio, los satélites de 226 kg que funcionan con energía solar se comunican entre sí por medio de enlaces ópticos y de radio. La red completa va a estar conectada a terminales de usuarios en la Tierra que tienen la posibilidad de instalarse en cualquier parte con vistas al cielo. Con un grupo de satélites lo bastante enorme sobre nuestras propias cabezas, el servicio de Internet debe estar disponible de manera continua, a diferencia de los retrasos de conectividad usuales en los recientes satélites Iridium y otras redes (Fox, 2001).

\section{Latencia}

Otra de las cuestiones que acostumbran hacer frente las conexiones satelitales es la de la latencia, en otros términos, la suma de retardos temporales de los paquetes que circularán por dicha red. Aquí Musk ha repetido en varias situaciones que debido a la baja órbita de dichos satélites no puede haber inconvenientes en este entorno, y por cierto promete latencias de $20 \mathrm{~ms}$-inferiores a las de varias conexiones recientes de fibra o ADSL_ que van a poder ser inclusive rebajadas a tan solo 8 ms en una segunda generación de Starlink en el futuro (Duan \& Dinavahi, 2021; Mar Cornelio et al., 2021; Omar Mar Cornelio, 2020).

\section{Metodología}

Para la elaboración del presente artículo, se puso en práctica los siguientes métodos:

Métodos empíricos:

Observación y experimentación: permitió generar antecedentes en la información, y de una u otra manera observar de manera clara la realidad del problema planteado.

Inductivo-deductivo: se basa en la lógica y el razonamiento, con este método pudimos obtener las conclusiones, ya que, se basa en la observación personal, hace comparaciones y analiza los hechos o características del tema.

Método de análisis-síntesis: este método consiste en analizar de forma particular el tema, estudiar y sacar hipótesis del mismo, en pocas palabras este método se encarga de recopilar información de forma individual de cada parte involucrada en el tema Impacto del internet global "Starlink" para obtener un buen resultado en cuanto a contenido.

Método objetivo-subjetivo: este método se basa en la subjetividad, en la realidad o lo positivo del tema, con este método pudimos obtener los objetivos de nuestra investigación, ya que nos hace creer o pensar más allá de la realidad física, con el método histórico -lógico, se hizo la

18 UNESUM-Ciencias. Publicación cuatrimestral. Vol. 6, Año 2022, No. 1 (Enero - Abril) 
construcción de nuestra investigación y determinamos las tendencias del objetivo, con acontecimientos relevantes antes sucedidos plasmados en el desarrollo del contenido y por último el método bibliográfico, se obtuvo una mejor selección de información, datos y material, proveniente de los diferentes sitios web, libros, revistas, etc. Los cuales fueron aportes fundamentales en nuestro artículo.

Entre los materiales utilizados se encuentran el internet, computadora de escritorio, laptops, teléfonos celulares, distintos sitios webs, libros, revistas científicas, etc.

\section{RESULTADOS Y DISCUSIÓN}

El internet satelital "Starlink" conectará al mundo mediante su inmensa red de satélites alrededor de la tierra que proporcionara internet de banda ancha a todo el planeta, especialmente en los entornos rurales y lugares con difícil acceso a las redes fijas o redes móviles. Este servicio no pretende competir con las conexiones 5G o la fibra que son las redes disponibles en los actuales momentos si no complementar ambas, Starlink no se basa en la velocidad si no en la disponibilidad del internet que a su vez cuenta con una latencia de $20 \mathrm{Ms}$ en la actualidad, pero que llegará a conseguir una latencia de 8Ms en la segunda generación de Starlink.

$\mathrm{Al}$ poner en órbita 42.000 satélites se generar una gran cantidad de desechos espaciales sin contar con las colisiones que se podrían presentar al tener esa cantidad de satélites en la órbita terrestre, pero Elon Musk en un comunicado dispuso que sus satélites están diseñados para no sobrevivir al entrar en la atmosfera de la tierra, también mencionó que sus satélites serán invisibles y que no afectará a la astronomía.

Las velocidades del servicio de internet Starlink han marcado una gran diferencia con otras empresas dentro del mundo satelital, pero cabe mencionar que SpaceX también tienes sus competidores como la empresa británica de telecomunicaciones y Blue Origin, sin embargo Starlink tiene grandes logros dentro del mundo.

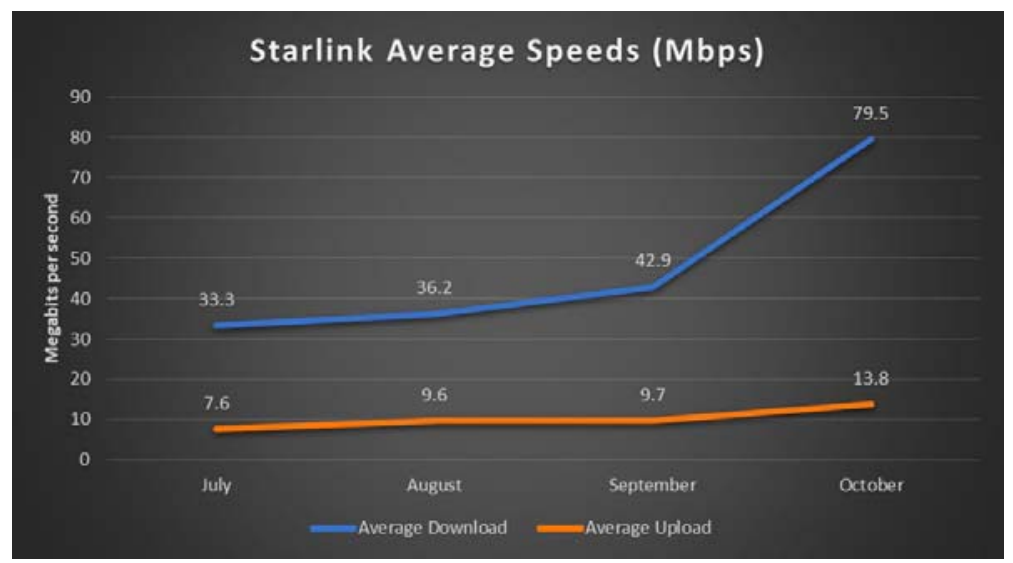

Figura 1. Velocidad del servicio de Internet satelital de Starlink de SpaceX.

La figura 1 muestra como las velocidades de Starlink se han inclinado bruscamente hacia arriba en los últimos meses a medida que el proyecto se acerca al lanzamiento. 


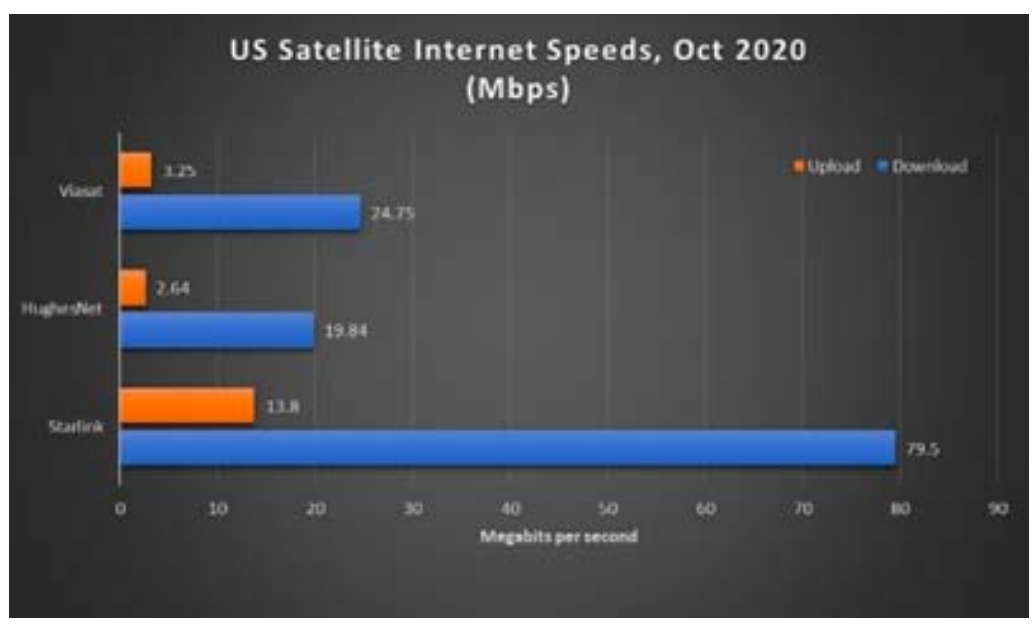

Figura 2. Velocidades de Starlink respecto HughesNet.

La figura 2 muestra como las velocidades de Starlink superan a Exede y HughesNet.

\section{CONCLUSIONES}

El internet Starlink tiene una perspectiva diferente a lo cotidiano, busca la disponibilidad de llegar sitios alejados donde nunca antes existía dicha oportunidad de acceder al internet, cabe recalcar que la velocidad de dicho internet no es su punto fuerte, pero cuenta con Gigabits para las velocidades de la transferencia de datos de descarga y una latencia que aproximadamente esta entre 7 y $30 \mathrm{~ms}$.

Actualmente es sabido que los avances dentro de la ciencia y tecnología aeroespaciales cada vez más han desarrollado diversas herramientas, lo que esto ayudaría mucho más a la constelación Starlink. Esto proporcionó excelentes resultados y logró una capacidad de persistencia activa, donde se pueden conectar miles de millones de usuarios a su red.

\section{REFERENCIAS BIBLIOGRÁFICAS}

Bratspies, R. M. (2002). Myths of voluntary compliance: lessons from the StarLink corn fiasco. Wm. \& Mary Envtl. $\begin{array}{llllll}\text { L. \& Pol'y Rev., } & 593 .\end{array}$ https://academicworks.cuny.edu/cgi/viewcontent.cgi?article=1236\&context=cl_pubs

Bromberg, Y.-D., Grace, P., \& Réveillere, L. (2011). Starlink: runtime interoperability between heterogeneous middleware protocols. 2011 31st International Conference on Distributed Computing Systems,

Chowdhury, E., Mikami, O., Nakajima, Y., Hino, A., Kuribara, H., Suga, K., Hanazumi, M., \& Yomemochi, C. (2003). Detection of genetically modified maize DNA fragments in the intestinal contents of pigs fed StarLink CBH351. Veterinary and Human Toxicology, 45(2), 95-96. https://europepmc.org/article/med/12678298

Disney, M., \& Wallace, P. (1982). Starlink. Quarterly Journal of the Royal Astronomical Society, 23, 485. http://adsabs.harvard.edu/pdf/1982QJRAS..23..485D

Duan, T., \& Dinavahi, V. (2021). Starlink Space Network Enhanced Cyber-Physical Power System. IEEE Transactions on Smart Grid. https://ieeexplore.ieee.org/abstract/document/9383100/

Fox, J. L. (2001). EPA re-evaluates StarLink license. Nature Biotechnology, 19(1), 11-11. https://www.nature.com/articles/nbt0101_11

Kassas, Z. Z., Khalife, J., \& Neinavaie, M. (2021). The first carrier phase tracking and positioning results with Starlink LEO satellite signals. IEEE Transactions on Aerospace and Electronic Systems.

20 UNESUM-Ciencias. Publicación cuatrimestral. Vol. 6, Año 2022, No. 1 (Enero - Abril) 
Mar Cornelio, O., Gulín González, J., \& Santana Ching, I. (2021). Sistema de Laboratorios Remoto para las prácticas de control de la carrera de Ingeniería en Automática. Revista Cubana de Ciencias Informáticas, 15(2), 7795.

McDowell, J. C. (2020). The low earth orbit satellite population and impacts of the SpaceX Starlink constellation. The Astrophysical Journal Letters, 892(2), L36. https://iopscience.iop.org/article/10.3847/20418213/ab8016/pdf

Omar Mar Cornelio, J. G. G., Ivan Santana Ching, Barbara Bron Fonseca. (2020). Remote Laboratory System for Automatic Engineering. International Journal of Wireless and Ad Hoc Communication, 1(2), 55-63.

Segarra, A. E., Rawson, J. M., Resources, S., \& Division, I. (2001). StarLink [TM] Corn Controversy: Background.

YONEMOCHI, C., FUJISAKI, H., HARADA, C., KUSAMA, T., \& HANAZUMI, M. (2002). Evaluation of transgenic event CBH 351 (StarLink) corn in broiler chicks. Animal Science Journal, 73(3), 221-228. 
22 UNESUM-Ciencias. Publicación cuatrimestral. Vol. 6, Año 2022, No. 1 (Enero - Abril) 